\title{
p190A RhoGAP is involved in EGFR pathways and promotes proliferation, invasion and migration in lung adenocarcinoma cells
}

\author{
HIROTSUGU NOTSUDA $^{1}$, AKIRA SAKURADA ${ }^{1}$, CHIAKI ENDO $^{1}$, YOSHINORI OKADA ${ }^{1}$, \\ AKIRA HORII $^{2}$, HIROSHI SHIMA ${ }^{3}$ and TAKASHI KONDO ${ }^{1}$ \\ ${ }^{1}$ Department of Thoracic Surgery, Institute of Development, Aging and Cancer, Tohoku University, Sendai 980-8575; \\ ${ }^{2}$ Department of Molecular Pathology, Tohoku University School of Medicine, Sendai 980-8574; \\ ${ }^{3}$ Division of Cancer Chemotherapy, Miyagi Cancer Center Research Institute, Natori 981-1293, Japan
}

Received May 23, 2013; Accepted July 2, 2013

DOI: 10.3892/ijo.2013.2096

\begin{abstract}
Overcoming acquired resistance to epidermal growth factor receptor tyrosine kinase inhibitors (EGFR-TKIs) is an emerging issue in lung cancer treatment. We report evidence that a GTPase-activating protein, p190-A RhoGAP (p190), is a potential molecular target for the treatment of lung adenocarcinoma. We documented inhibition of phosphorylation of p190 by EGFR-TKI treatment in lung adenocarcinoma cell lines. Small interfering RNA-mediated knockdown of p190 leads lung adenocarcinoma cells to growth suppression and to inhibition of invasion/migration through inducing cell cycle arrest but not apoptosis. These findings were observed not only in EGFR-TKI-sensitive cells but also in EGFR-TKIresistant cells; even in cell lines harboring K-ras mutations. The mechanism of this inhibitory effect on growth and invasion/migration was Ras inactivation through disrupting the p190-A RhoGAP/p120RasGAP complex. In addition, a high level of p190 mRNA expression was observed in majority of surgically obtained tissue from lung adenocarcinoma patients. Overexpression of p190 mRNA associated with poor diseasefree survival. The results suggest that overexpression of p190 mRNA may be involved in the carcinogenesis of lung adenocarcinoma. These findings indicate that p190 is a possible molecular target for treatment of lung adenocarcinoma.
\end{abstract}

\section{Introduction}

Lung cancer is the leading cause of cancer-related mortality worldwide, with a 5-year survival rate of only $20 \%$. Although patients diagnosed with early-stage disease have the potential

Correspondence to: Dr Akira Sakurada, Depertment of Thoracic Surgery, Institute of Development, Aging and Cancer, Tohoku University, 4-1 Seiryomachi, Aoba-ku, Sendai 980-8575, Japan

E-mail: sakurada@idac.tohoku.ac.jp

Key words: p190-A RhoGAP, phosphorylation, EGFR, lung cancer, therapeutic target for cure with complete surgical resection, only $25 \%$ of lung cancer cases are diagnosed at an early stage.

Small molecule inhibitors of epidermal growth factor receptor tyrosine kinase (EGFR-TKI), such as gefitinib and erlotinib $(1,2)$, induce dramatic responses in certain patients with non-small cell lung cancer (NSCLC) (3). EGFR mutation (short deletion in exon 19, L858R) positive patients have shown an impressive $60 \%$ response rate, which exceeds the response rate for conventional chemotherapy (4). However, the clinical success of treatment with EGFR-TKIs is uniformly limited by the development of acquired drug resistance. Two major mechanism of acquired resistance have been identified as T790M mutation in EGFR gene and gene amplification of MET oncogene $(5,6)$. Hence, new therapeutic strategies, such as the development of more effective or alternative moleculartargeted agents against lung cancer are eagerly awaited.

Recently, large-scale screening methods have been applied to identify novel molecular targets. Mass spectrometry targeting phosphorylated tyrosin on lung adenocarcinoma cells harboring EGFR kinase domain mutation has been reported and revealed that phosphorylation of several proteins, which have not been identified in EGFR pathway, is strongly controlled by EGFR protein (7). We performed similar analysis and found that p190-A RhoGAP (p190) was one of common proteins which include functional tyrosine controlled by EGFR protein.

p190 is a $172-\mathrm{kDa}$ protein that is encoded by the GRLF1 gene located on 19q13.3. The direct action of p190 has been reported to be a RhoGAP; converting Rho-GTP to Rho-GDP and inactivating the Rho pathway (8). Therefore, p190 has been thought to be a potent inhibitor of RhoA (9). On the other hand, indirect action of p190 has been reported to control the Ras pathway by binding to p120RasGAP protein. p190 has a binding activity to $\mathrm{p} 120 \mathrm{RasGAP}$ and the activity is promoted by phosphorylation of tyrosine $\left(\mathrm{Y}^{1105}\right)$ of p190 (10). The activity of p120RasGAP is reduced when p190 forms a complex with p120RasGAP (11). So far, p190 has been reported to inhibit cell migration and invasion through negatively regulating RhoA in some cell lines (12). On the other hand, EGF-induced p190 phosphorylation has been reported to play essential roles in Ras activation and cell proliferation using different cell lines (13). To date, only few reports are available on the function of 
p190 in lung cancer. We investigated the roles of p190 in lung adenocarcinoma cells and clarified whether p190 could be a potential therapeutic target for lung cancer.

\section{Materials and methods}

Cell lines and culture. A549, H520 and H1975 were obtained from American Type Culture Collection; ATCC (Manassas, VA, USA). PC-14 was obtained from DS Pharma Niomedical Co., Ltd. (Suita, Osaka, Japan). LK87, LCSC\#1 and II-18 were obtained from Cell Resource Center for Biomedical Research (Tohoku University, Sendai, Japan). All cells were maintained in RPMI-1640 (Invitrogen, San Diego, CA, USA) supplement with $10 \% \mathrm{FBS}$ in a humidified $5 \% \mathrm{CO}_{2}$ incubator at $37^{\circ} \mathrm{C}$. A549 and LK87 have wild-type (WT) EGFR and K-ras mutation (codon 12), PC-14 has EGFR mutation (exon 19 del), LCSC\#1 and II-18 have EGFR mutation (L858R) and H1975 has EGFR mutation (L858R and T790M). H520, with squamous cell carcinoma histology, has both WT EGFR. A549, H1975 and H520 have been reported to be EGFR-TKI-resistant, whereas LCSC\#1, PC-14 and II-18 to be EGFR-TKI-sensitive. NHBE (normal human bronchial epithelial cell) was obtained from Takara Bio Inc. (Otsu, Shiga, Japan) and was grown in BEGM Bullet kit (Lonza Walkersville Inc., Walkersville, MD, USA) according to the manufacturer's instructions.

Western blot and immunoprecipitation analyses. Protein samples were obtained from cells and transferred onto an Immune-Blot PVDF Membrane (Bio-Rad, Hercules, CA, USA). Proteins on the membranes were incubated with recommended concentration of primary antibodies, horseradish peroxidase-labeled secondary antibodies and were visualized on ImageQuant LAS 4000 mini (GE Healthcare, Buckinghamshire, UK) by means of the ECL Western blot detection system.

For immunoprecipitation, the p190-A RasGAP antibody was bound on to Protein A Dynabeads ${ }^{\circledR}$ (Invitrogen Dynal, Oslo, Norway). The Protein A Dynabeads-p190-A RasGAP complex was incubated with the cell lysates of lung cancer cells. Obtained bead-protein complexes were eluted and analyzed by SDS-PAGE and western blotting. Antibodies to EGFR, phospho-EGFR, p190, phoshotyrosine, Src, phospho-Src family kinase, MEK, phospho-MEK, Erk, phospho-ErK and $\beta$-actin were purchased from Cell Signaling Technology, whereas antibodies to Ras, Rho and p120 were from Abcam.

RNA interference (RNAi). Cells were transfected with $5 \mathrm{nM}$ siRNA directed to p190-A RhoGAP (p190 siRNA; Silencer ${ }^{\circledR}$ Select Validated siRNA, Applied Biosystems, Austin, TX, USA) or negative control siRNA (NR siRNA; non-related siRNA, Applied Biosystems) using the Lipofectamine RNAiMAX $^{\circledast}$ (Invitrogen), according to the manufacturer's instructions. The target sequence of siRNA for p190 was GGCUGAUGUUGAUCUGCGATT.

cDNA synthesis and real-time PCR. cDNA synthesis using TaqMan ${ }^{\circledR}$ Gene Expression Cells-to-Ct ${ }^{\mathrm{TM}}$ kits was performed according to the manufacturer's instructions. Semi-quantitative real-time RT-PCR was performed by using the ABI PRISM 7000 HT sequence detection system (Applied Biosystems).
The Assays-on-Demand products purchased from Applied Biosystems contained TaqMan minor groove binder probes (6-FAM dye-labeled) combined with the primers for $\mathrm{p} 190$ (Hs00534180_m1). An Assay-on-Demand product for 18s-rRNA (4319413E) was used as endogenous control.

Cell proliferation, migration and invasion assays. Cell proliferation assay was performed using CellTiter $96^{\circledR}$ AQueous One Solution Cell Proliferation assay kit (Promega, Madison, WI, USA), according to the manufacturer's instructions. Transwell migration assay using CytoSelect ${ }^{\mathrm{TM}}$ 24-well Cell Migration assay (Cell Biolabs, San Diego, CA, USA) and Transwell invasion assay using CytoSelect ${ }^{\mathrm{TM}}$ 24-well Cell Invasion assay (Cell Biolabs) were performed according to the manufacturer's instructions.

Cell cycle analysis. Cells were fixed with $70 \%$ ice coldethanol and stored at $-20^{\circ} \mathrm{C}$. Then cells were incubated with $0.5 \%$ RNAse followed by propidium iodide. Histograms were obtained by FACSCanto II (BD). Doublets were excluded by scattergrams. Data were analyzed using ModFit software.

Quantitative assay for GTP-bound Ras and GTP-bound Rho. The levels of GTP-bound Ras and GTP-bound Rho were detected with the Raf-1 Ras binding domain agarose (Cell Biolabs) and GST-rohtekin pull-down assays (Cell Biolabs), respectively.

Real-time PCR on clinical samples. A total of 133 cDNA samples of pulmonary adenocarcinomas (pathological stage IA-IIIB) and adjacent normal lung tissue, were obtained along with clinicopathological data from patients who had undergone surgery at the Miyagi Cancer Center or Tohoku University Hospital. Quantitative RT-PCR was performed by an ABI 7000 using TaqMan probes for p190. $\beta$-actin was used as an internal control. Threshold cycles of prier probes were normalized to $\beta$-actin and translated to relative values.

Immunohistochemical analysis. Expression of phospho-p190 protein in paraffin-embedded clinical tissues was examined using the Linked Streptavidin-Biotin method (Histofine SAB-PO, Nichirei Biosciences, Japan). Rabbit anti-phosphop190 antibody (1:50 dilution; Abcam) was used as a primary antibody. Two independent investigators evaluated the immunohistochemical staining without knowledge of the clinicopathological parameters. For semi-quantitative assessment of the immunohistochemical results, the mean percentage of positive tumor cells was determined in $\geq 10$ random fields at $\mathrm{x} 400$ magnification in each section. It was graded as focal $(<10 \%)$, regional $(11-50 \%)$, or diffuse $(>50 \%)$. The intensity of the phospho-p190 immunoreaction was graded weak, moderate or strong. The mean percentage of positive tumor cells and the staining intensity were then combined to produce a phospho-p190-IHC result.

Survival and statistical analyses. Two-year disease-free survival (2-year DFS) was calculated from the time of surgery to the patient's last follow-up or recurrence. DFS of patients was analyzed using Kaplan-Meier survival curves and the logrank test was used to examine the statistical significance. All 


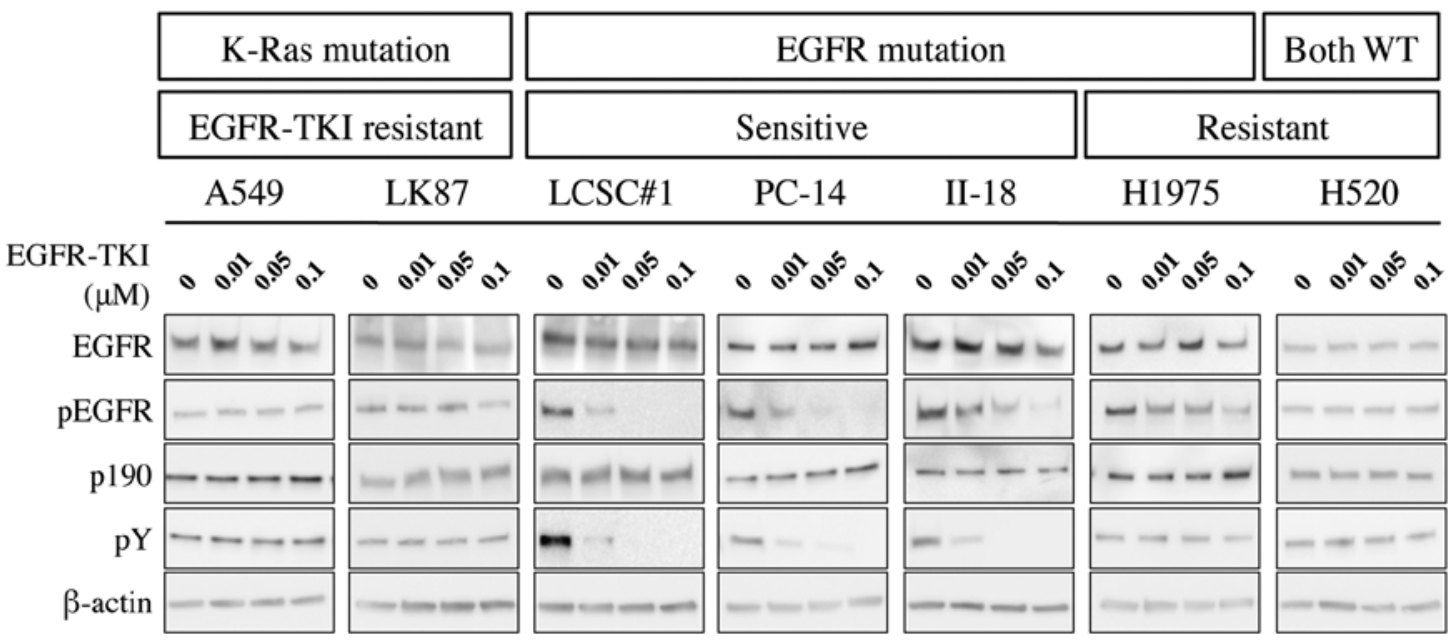

Figure 1. Results of western blot analysis and immunoprecipitation of p190 and EGFR in lung adenocarcinoma cell lines. Phosphorylation of EGFR at $\mathrm{Y}^{1068}$ is inhibited by EGFR-TKI treatment in EGFR-TKI-sensitive cell lines; LCSC\#1, PC-14 and II-18, but is retained in EGFR-TKI-resistant cell lines; A549, LK87, H1975 and H520. Phosphorylation of p190 was parallel to that of EGFR. Cells were pretreated with 0-0.1 $\mu$ M EGFR-TKI for $3 \mathrm{~h}$ before stimulation by $50 \mathrm{ng} / \mathrm{ml}$ epidermal growth factor (EGF) for $10 \mathrm{~min}$.

in vitro experiments were performed in triplicate. Comparisons between 2 groups were carried out using unpaired Student's t-test; comparisons among 3 or more groups were carried out using one-way ANOVA. Survival and statistical analysis were carried out with the Prism 5 for Mac OS X (GraphPad Software, USA). All P-values $<0.05$ were considered as statistically significant.

\section{Results}

Western blotting showed that phosphorylation of EGFR was inhibited by treatment with EGFR-TKI in EGFR-TKI-sensitive cells; LCSC\#1, PC-14 and II-18, but not in EGFR-TKI-resistant cells; A549, LK87, H1975 and H520. Similarly, immunoprecipitation analysis showed that phosphorylation of p190 was inhibited by administration of EGFR-TKI (Fig. 1).

Since no chemical inhibitor for p190-tyrosine phosphorylation is currently available, we performed p190 knockdown using siRNA (p190 RNAi). Before knockdown, we confirmed that all lung cancer cell lines in this study show high levels of p190 mRNA expression by RT-PCR, 20-160-fold higher than that of NHBE (Fig. 2A). To test the effect on cell proliferation after p190 RNAi, MTS assay was performed. The results showed that cellular proliferation of all lung adenocarcinoma cell lines in this study was significantly suppressed by p190 RNAi, while such suppression was not observed in H520 and NHBE (Fig. 2B).

To understand the mechanism of the growth suppression we further performed cell cycle analyses using lung cancer cells after p190 RNAi. A decrease in the S population was observed after p190 RNAi in all lung adenocarcinoma cell lines but not in the squamous cell carcinoma H520 (Fig. 3). These results indicate that p190 RNAi led the lung adenocarcinoma cells to cell cycle arrest.

p190 is a RhoGAP, and reportedly is involved in control of cell invasion and migration. To clarify this function in lung cancer cell lines, we knocked down p190 and tested invasion
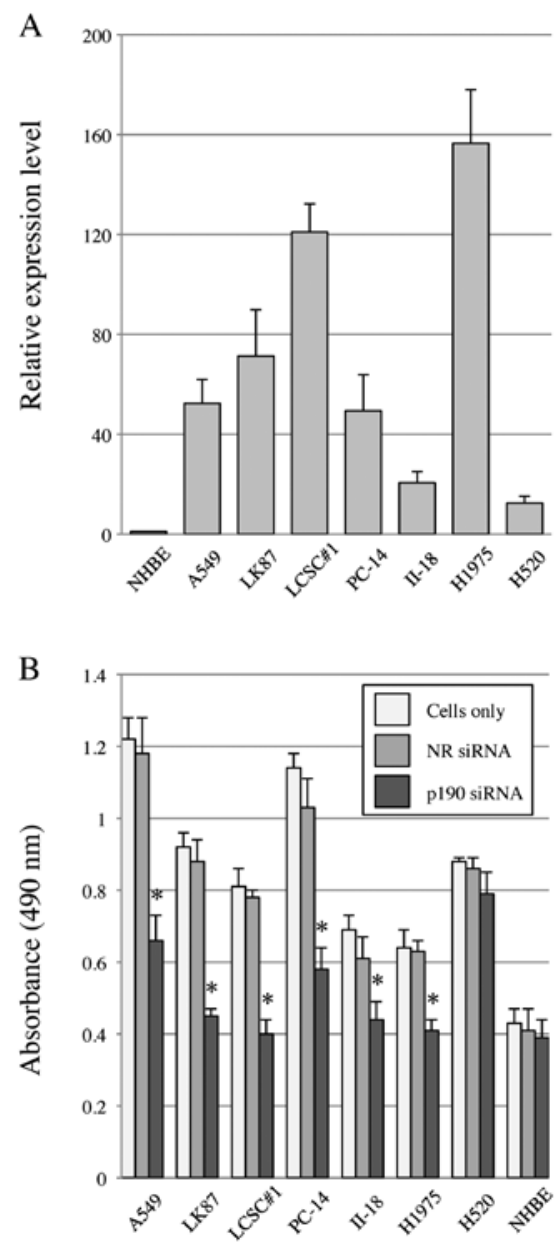

Figure 2. Results of cell proliferation assays after p190 knockdown using siRNA (p190 RNAi) in lung adenocarcinoma cell lines. (A) Relative expression level of p190 mRNA in lung adenocarcinoma cell lines. The expression relative to normal human bronchial epithelial cells (NHBE) is significantly elevated in all lung cancer cell lines. (B) Results of MTS assays after p190 RNAi. Bar graph depicts cell proliferation (measured by SpectraMax M2e) after 3 days of growth in lung cancer cell lines following p190 RNAi. The proliferation of all lung adenocarcinoma cell lines is inhibited by p190 RNAi. A paired t-test identified statistically significant difference in cell growth $\left({ }^{*} \mathrm{P}<0.05\right)$. Error bars represent $\mathrm{SD}$. 

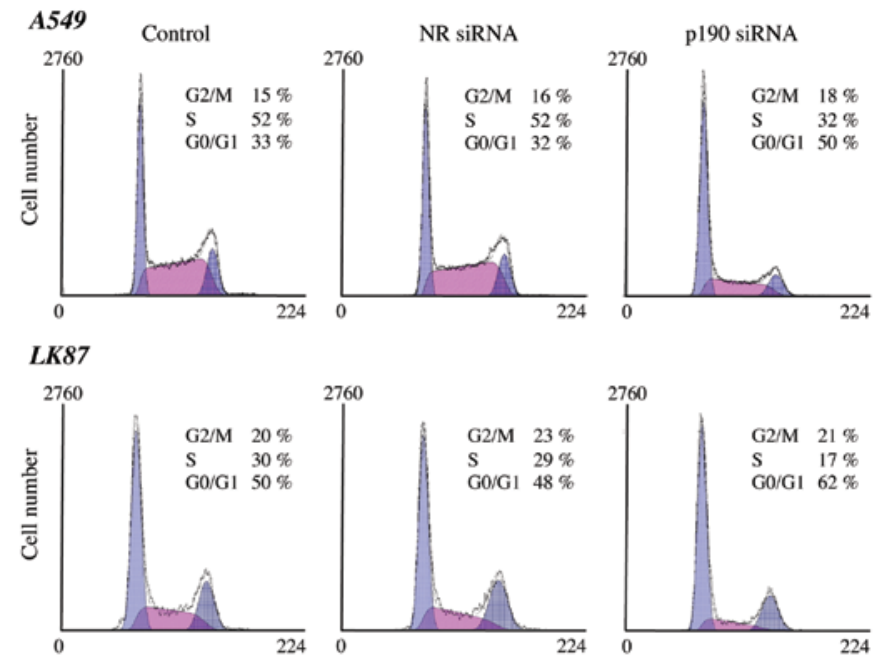

\section{LCSC\#I}
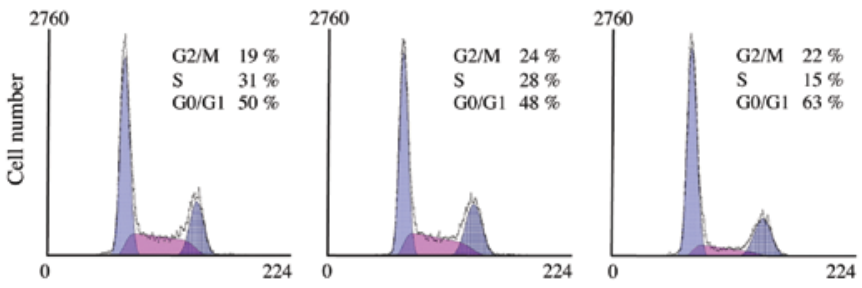

\section{PC-14}
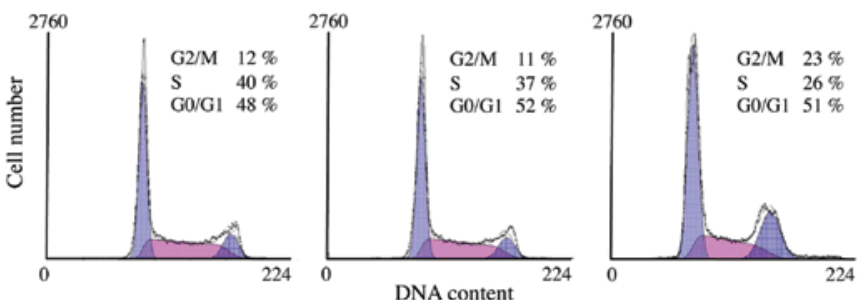
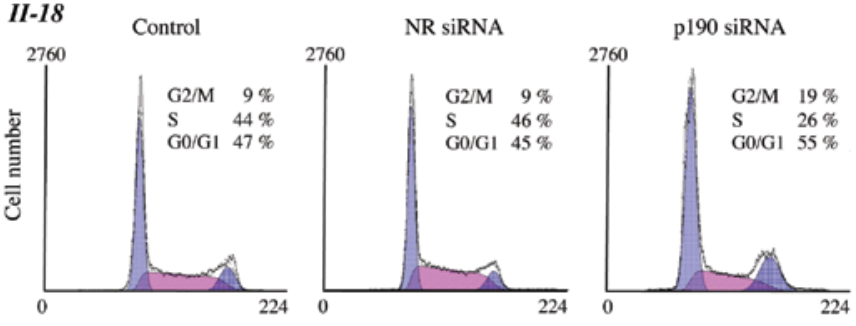

$\mathrm{H1975}$
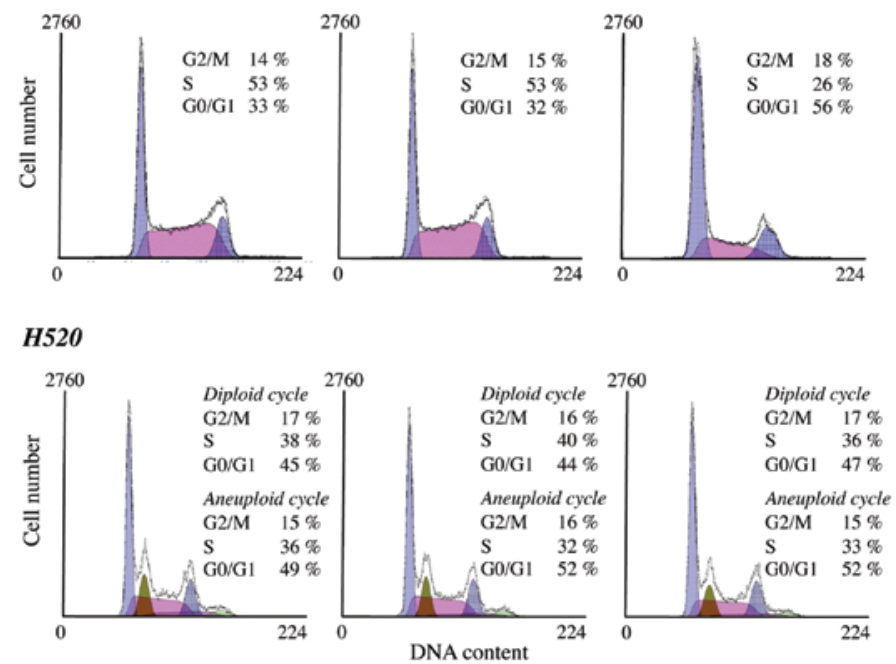

Figure 3. Results of cell cycle analysis of lung cancer cell lines. After 72-h siRNA treatment, cells were stained with propidium iodide (PI) and analyzed by flow cytometry. Percentages of cells in $\mathrm{G} 0 / \mathrm{G} 1, \mathrm{~S}$ and $\mathrm{G} 2 / \mathrm{M}$ population are shown in the right corner of each histogram. Cell cycle arrest occurs in all adenocarcinoma cell lines by p190 RNAi. and migration of lung cancer cells with high levels of p190 mRNA. By p190 RNAi, both cell invasion and migration were significantly reduced in A549, LK87, LCSC\#1, PC-14 and H1975 (Fig. 4). In II-18 cell and H520, the difference was not detected probably due to original nature of low activity of cell invasion/migration.

Phosphorylation of $\mathrm{p} 190$ at $\mathrm{Y}^{1105}$ is known to promote formation of a p190/p120 complex. Formation of the complex has been reported to downregulate the RasGAP activity of p120, leading to an elevation of Ras activity. These complexes upregulate the RhoGAP activity of p190, leading to a repression of Rho activity. We investigated the effect of both EGFR-TKI and p190 RNAi on the p190/p120 complex formation, Ras activation and Rho inactivation in lung cancer cell lines. Immunoprecipitation analysis showed that p190/ p120 complex formation was inhibited in EGFR-TKI-sensitive cell lines by EGFR-TKI treatment in a dose-dependent manner, but not in EGFR-TKI-resistant cell lines. On the other hand, formation of the complex was inhibited by p190 RNAi in all lung cancer cell lines (Fig. 5A). Ras inactivation was parallel to disruption of p190/120 complex by EGFR-TKI or p190 RNAi in all adenocarcinoma cell lines, while Rho activation was inverse to disruption of p190/120 complex in the adenocarcinoma cell lines except II-18 (Fig. 5B). In II-18 cells, RhoA activation could not be evaluated due to low internal protein level of RhoA.

Phosphorylation of MEK and Erk, downstream molecules of Ras, were inhibited by p190 RNAi in all adenocarcinoma cell lines, although the effect is relatively small in A549. In contrast, phosphorylation of Src, a potential upstream molecule of p190, was inhibited by EGFR-TKI in EGFR-TKI-sensitive cells but not in EGFR-TKI-resistant cells, while no effect of p190 RNAi was observed (Fig. 6).

We also studied the expression level of p190 in clinical samples. Relative expression level of p190 mRNA in tumor to paired normal lung tissue was tested by RT-PCR in 133 human lung adenocarcinoma tissue samples (Table I). High level of p190 mRNA was detected in majority of samples of lung adenocarcinoma. Pathological stage III patients $(n=18)$ had significantly higher mRNA level of p190 compared with stage IA or IB patients $(\mathrm{P}<0.05)$ (Fig. 7A). Whereas EGFR mutation existed in 56 samples, there was no association between p190 mRNA level and EGFR mutation of the tumors $(\mathrm{P}=0.703)$ (Fig. 7B). Furthermore, we analyzed a cohort of 133 patients with outcome data. By ROC curve analysis, we determined a threshold of p190 mRNA expression level as 

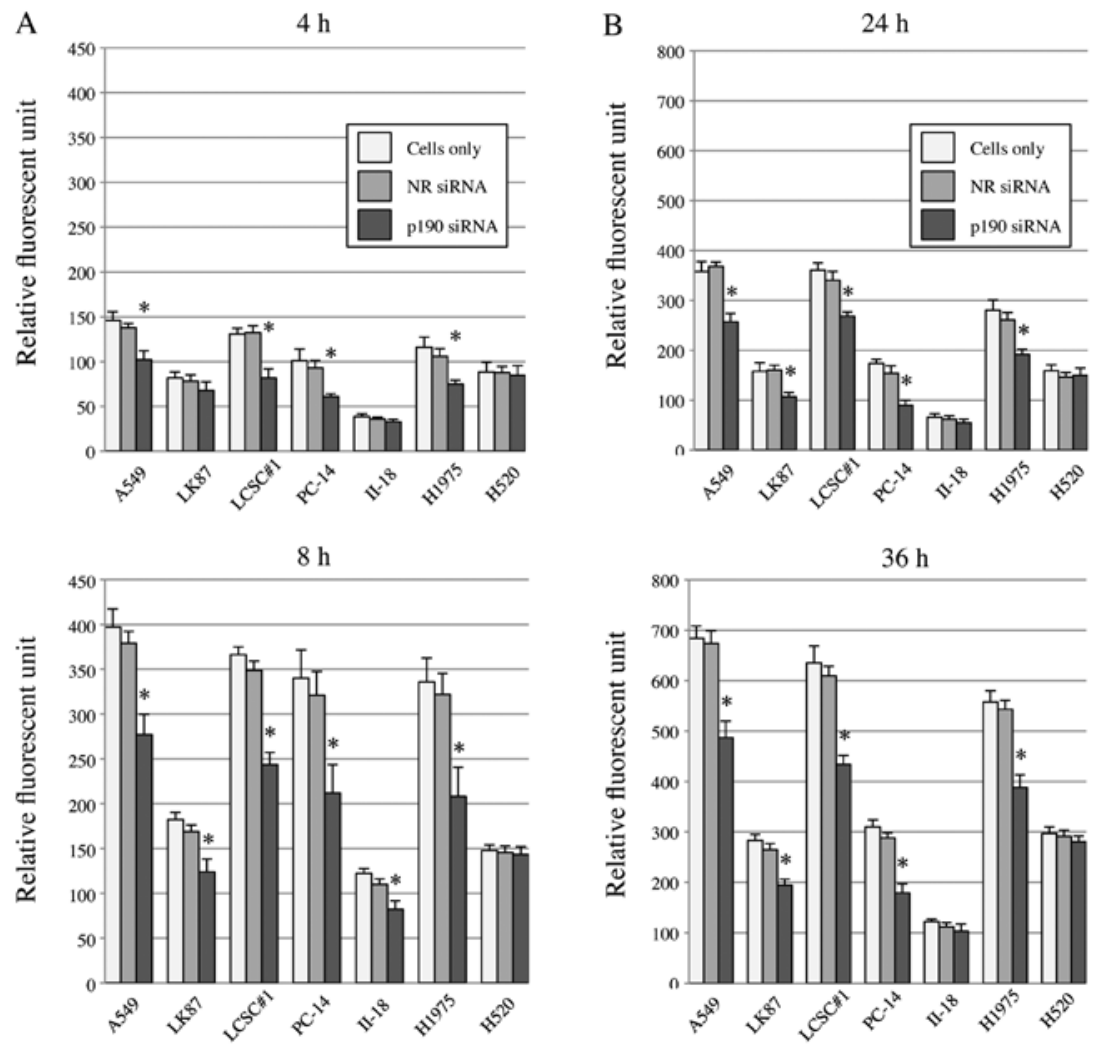

Figure 4. Results of cell invasion and migration assays after p190 RNAi in lung adenocarcinoma cell lines. (A) Results of migration assays. After 24-h siRNA treatment, cell suspension was placed in the upper chamber. Four or $8 \mathrm{~h}$ later migratory cells that passed through polycarbonate membrane were lysed and quantified using fluorescent dye (measured by SpectraMax M2e). The migration of A549, LK87, PC-14 and H1975 are inhibited by p190 RNAi. A paired t-test identified statistically significant difference in cell growth ("P<0.05). (B) Results of invasion assays. Similar to the migration assays, the invasion of A549, LK87, PC-14 and H1975 are inhibited by p190 RNAi.

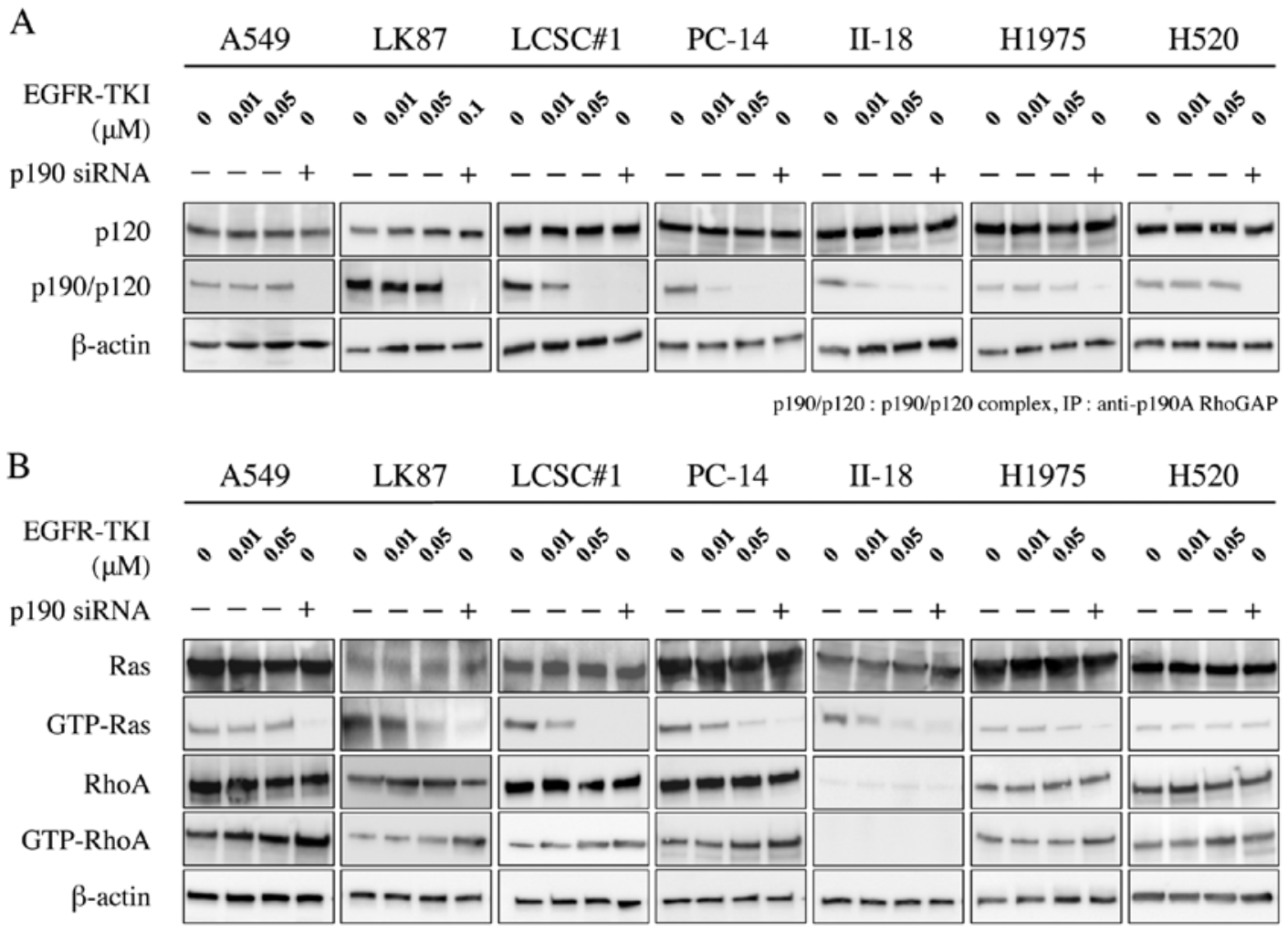

Figure 5. Western blot and immunoprecipitation analyses of Ras and Rho signal pathways in lung adenocarcinoma cell lines. (A) Results of immunoprecipitation with p120/p190 complex. p120 are not detected by western blotting after p190 RNAi in the lung adenocarcinoma cell lines. (B) Results of Ras and Rho activation assay. GTP-bound active form Ras is not detected after p190 RNAi in the lung adenocarcinoma cell lines or after administration of EGFR-TKI in PC-14 and II-18. GTP-bound active form Rho is detected after p190 RNAi in all the lung cancer cell lines, except II-18. 


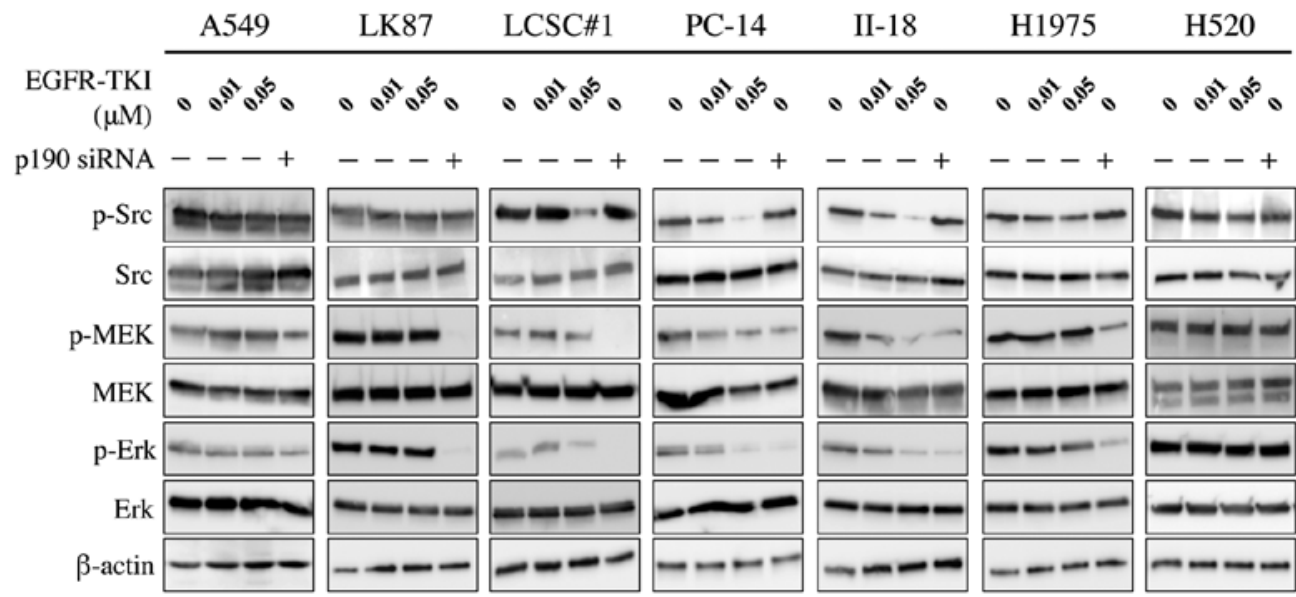

Figure 6. Western blot analysis of Src, MEK, Erk and phosphorylation. Src is predicted to be upstream of p190 in EGFR signaling pathway. Phosphorylation of Src is induced in the lung adenocarcinoma cell lines regardless of p190 RNAi. MEK and Erk are predicted to be downstream of p190 in EGFR signaling pathway. Phosphorylation of MEK and Erk is inhibited in all the cell lines by p190 RNAi.

Table I. Characteristics of the 133 lung adenocarcinoma patients whose surgical specimens were studied by RT-PCR.

\begin{tabular}{lc}
\hline Characteristics & No. of patients $(\%)$ \\
\hline Age at surgery & $67(50)$ \\
$<70$ & $66(50)$ \\
$\geq 70$ & \\
Gender & $64(48)$ \\
Male & $69(52)$ \\
Female & \\
Pathological stage & $64(48)$ \\
IA & $38(29)$ \\
IB & $13(10)$ \\
II & $18(13)$ \\
III & \\
EGFR mutation & \\
Positive & $56(43)$ \\
L858R & $22(17)$ \\
Exon19 deletion & $30(23)$ \\
Other mutations & $4(3)$ \\
Negative & $77(57)$ \\
p190 relative expression level & \\
$<3$ & $79(59)$ \\
$\geq 3$ & $54(41)$ \\
\hline & \\
& \\
&
\end{tabular}

three times the level of corresponding normal tissue of the patients. High expression of p190 is associated with poor disease-free survival ( $\mathrm{P}=0.003)$ (Fig. 7C).

Phosphorylated-p190 (phospho-p190) was evaluated immunohistochemically in 52 adenocarcinoma specimens. Immunohistochemical staining showed that $92 \%$ (48 of 52) of pulmonary adenocarcinoma samples were positive for phospho-p190 (Table II). The staining intensity was also
Table II. Immunohistochemical staining of phospho-p190 protein in the 52 lung adenocarcinoma specimens.

\begin{tabular}{|c|c|c|c|c|}
\hline \multirow[b]{2}{*}{ Characteristics } & \multirow[b]{2}{*}{ Total no. } & \multicolumn{2}{|c|}{ No. IHC score } & \multirow[b]{2}{*}{ P-value } \\
\hline & & 0 or 1 & 2 or 3 & \\
\hline Age at surgery & & & & 0.092 \\
\hline$<70$ & 23 & 15 & 8 & \\
\hline$\geq 70$ & 29 & 11 & 18 & \\
\hline Gender & & & & 0.10 \\
\hline Male & 25 & 9 & 16 & \\
\hline Female & 27 & 16 & 11 & \\
\hline Pathological stage & & & & 1 \\
\hline $\mathrm{IA}, \mathrm{IB}$ & 41 & 21 & 20 & \\
\hline II-IV & 11 & 5 & 6 & \\
\hline EGFR mutation & & & & 0.78 \\
\hline Positive & 28 & 15 & 13 & \\
\hline Negative & 24 & 11 & 13 & \\
\hline \multicolumn{5}{|l|}{ p190 relative mRNA } \\
\hline level & & & & $<0.005$ \\
\hline$<3$ & 30 & 21 & 9 & \\
\hline$\geq 3$ & 22 & 5 & 17 & \\
\hline
\end{tabular}

stronger in tumor cells than that in normal cells (Fig. 8A). No statistically significant difference was found between IHC score and clinical outcome of lung adenocarcinoma patients (Table II). However, there were statistically significant association between histological features and phospho-p190 staining using Fisher's exact-test ( $\mathrm{P}=0.013)$ (Fig. 8B).

\section{Discussion}

In this study, we found three novel findings. First, we clearly demonstrated that phosphorylation of p190 is involved in the 
A
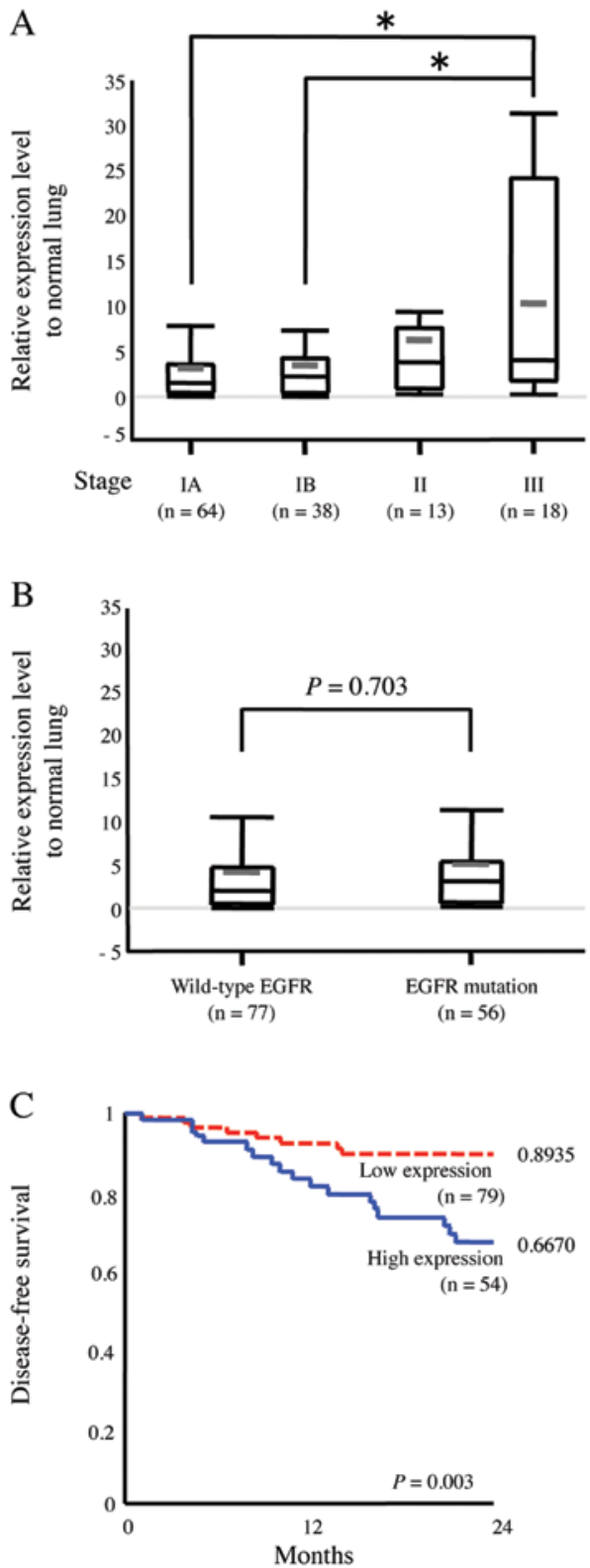

Figure 7. Relative expression level of p190 mRNA in human lung adenocacinoma specimens. Numbers indicate relative p190 mRNA expression normalized against $\beta$-actin. (A) Box plot of p190 mRNA expression levels in lung adenocarcinoma patients based on pathological stage IA/IB/II/III shows that average 190 expression correlates with pathological stage in lung adenocarcinoma. (B) Box plot of p190 mRNA expression levels in lung adenocarcinoma patients based on EGFR mutation status shows that average p190 expression does not correlate with EGFR mutation status of lung adenocarcinoma. There is no correlation between mRNA level and pathological stage of the patients. (C) Kaplan-Meier analysis shows that patients with high expression of p190 mRNA have a significantly poor disease-free survival probability $(\mathrm{P}=0.003)$.

EGFR signal pathway. Previous mass spectrometry screening suggested that tyrosine $\left(\mathrm{Y}^{1105}\right)$ phosphorylation of $\mathrm{p} 190$ is affected by EGFR-TKI. Here, we discovered that one point detected by previous mass spectrometry analysis is actually on the line of EGFR signaling pathway by detailed western blot analysis. We highlighted unique functional characteristics of p190RhoGAP, a sort of switch of Ras and Rho pathway and how EGFR-TKI affects this switch in lung adenocarcinoma cells. As briefly described in the introduction, 190 is one of the
A
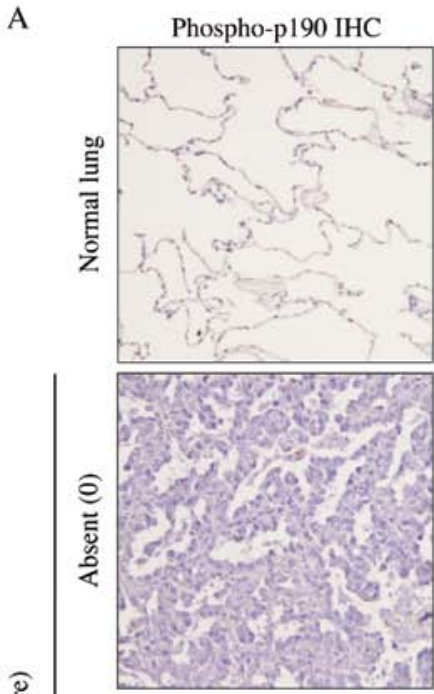

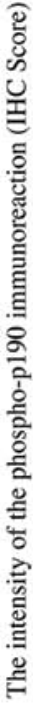
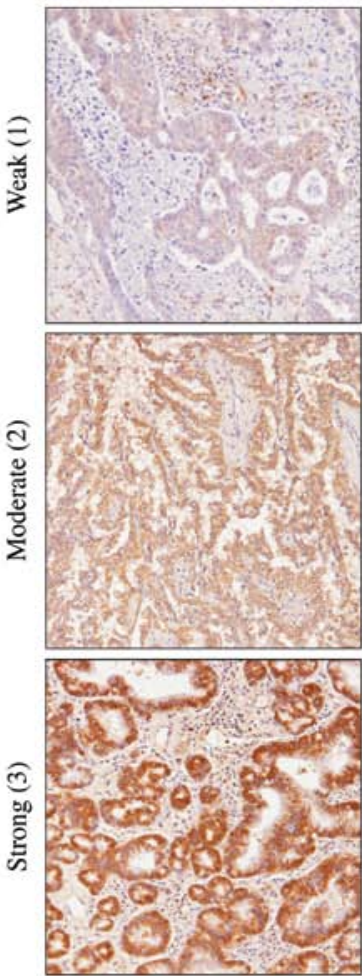

B

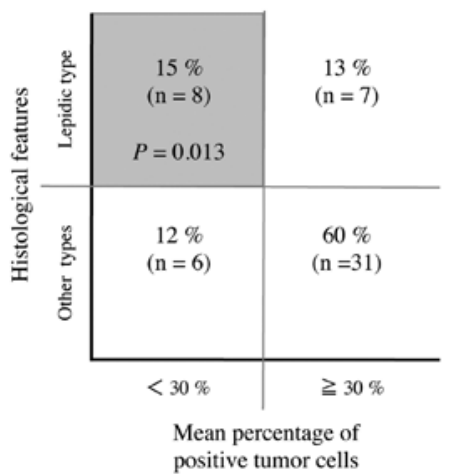

Figure 8. Immunohistochemical staining analysis of human lung adenocarcinoma specimens. (A) Representative photomicrograhs show phospho-p190 expression in normal lung and lung adenocarcinomas. IHC score is indicated in parenthesis. (B) Correlation analysis of histological features with the mean percentage of positive tumor cells in lung adenocarcinoma tumors $(n=52$ tumors). Gray box highlights a statistically significant association between histological features and the mean percentage of positive tumor cells using Fisher's exact-test $(\mathrm{P}=0.013)$. 
GTPase activating proteins (GAPs) and converts RhoA, small GTPases, from a GTP-bound active form into a GDP-bound inactive form (14). In addition, previous studies have reported that activated p190 positively regulates the Ras pathway, by forming complex with p120RasGAP which converts Ras-GTP to Ras-GDP $(8,10)$. We demonstrated that 190 played a crucial role in regulation of Ras and Rho in lung adenocarcinoma cells. Furthermore, we demonstrated that, by p190 RNAi, the same molecular behavior, such as Ras inactivation and Rho activation to EGFR-TKI was observed not only in EGFR-TKIsensitive cells but also in EGFR-TKI-resistant cells.

Second, we showed that cell proliferation as well as cell migration/invasion is significantly impaired by p190 RNAi in lung adenocarcinoma cell lines. Our results of cell cycle analysis revealed that suppression of proliferation in lung adenocarcinoma cells was caused by cell cycle arrest, not by apoptosis. Interestingly, arrest patterns, such as G1/S or G2/M was different in cells. At present, it is not possible to explain clearly the reason of the difference, underlying different molecular background between cells is possible reason. As previously reported, apoptosis is induced in lung adenocarcinoma cells harboring EGFR kinase mutation by treatment of EGFR-TKI (15). The p190ARhoGAP-Ras pathway is not responsible or is not enough for induction of apoptosis in EGFR-TKI-sensitive cells. Nevertheless, it is noteworthy that significant suppression of cell proliferation is observed by 190 RNAi in EGFR-TKI-resistant cells; even in A549 and LK87 harboring KRAS mutation which generates constitutivelyactive Ras protein (16).

By western blot analysis, we showed that RhoA protein was activated by p190 RNAi, while Ras protein is inactivated, thus discordant molecular signaling, both promoting and inhibiting cell proliferation, were generated simultaneously. Results of MTS assay indicates that Ras signaling rather than Rho signaling is predominant on cell proliferation in lung adenocarcinoma cells. KRAS mutation has been reported to exist in $\sim 20 \%$ of lung cancer patients and to be mutually exclusive with EGFR mutations (17). Although the mutations in EGFR correlate with sensitivity of the tumors to EGFRTKIs, K-ras mutations are associated with primary resistance to EGFR-TKIs (18). This result indicates that p190 is a promising molecular target for lung adenocarcinoma regardless of the mutation type of lung cancer.

Invasion and metastasis are the leading causes of treatment failure and the most significant predictor of poor clinical outcome in patients with lung cancer (19). Increased motility is the first step in invasion and metastasis. RhoA expression is associated with poor prognosis especially in breast cancer (20), hepatic carcinoma (21) and pancreatic cancer (22). Our study showed that GTP-binding active form RhoA existed in the six lung adenocarcinoma cell lines, except II-18 by p190 RNAi. On the basis of this result, we expected that ability of invasion and migration would be increased in lung adenocarcinoma cell lines by p190 RNAi. Whereas, our study disclosed that in vitro invasion/migration has been significantly inhibited in lung adenocarcinoma cells, except II-18. One possibility is that, in lung adenocarcinoma cells, cross-talk between Rho family of GTPases, such as RhoA, Rac1 and Cdc42 exists and control activation of cell invasion and migration $(23,24)$. Alternatively, cell invasion and migration might be affected by inactivation of Ras followed by cell cycle arrest (25).

Third, overexpression of p190 mRNA was detected from all lung adenocarcinoma cell lines. In addition, high expression of p190 mRNA significantly correlated to advanced pathological stage and was also associated with poor disease-free survival. Unexpectedly, EGFR mutation did not correlate with either mRNA level of p190 or immunostaining results of phospho-p190. This might suggest that p190 receives also other signals than the EGFR pathway in vivo. By immunohistochemical study, almost all (92\%) pulmonary adenocarcinoma specimens were positive for phospho-p190 protein. Overexpression of p190 mRNA was observed in the patients with early stage lung cancer, and phospho-p190 staining was observed in almost all patients of lung cancer suggesting that overexpression of p190 may be involved in early carcinogenesis of lung adenocarcinoma. Interestingly, lepidic type histology, which is generally recognized to show less invasive nature, showed negative correlation to expression of phospho-p190 protein.

In conclusion, phosphorylation of p190 is regulated by EGFR in lung adenocarcinoma cells and p190 involves cell proliferation and migration/invasion through controlling the Ras signaling pathway. Studies on clinical specimens suggest that p190 is associated with aggressive nature of the tumors. p190 shows promise as a novel molecular target for treatment of lung adenocarcinoma including tumors with acquired resistance to EGFR-TKI or with K-ras mutation.

\section{Acknowledgements}

The authors thank Drs Nobuhiro Tanuma, Hiroteru Miyashita, Takayuki Nakagawa, Hisashi Ohishi, Sumiko Maeda, Masafumi Noda, Tetsu Sado and Yasushi Hoshikawa for their technical assistance and advice.

\section{References}

1. Baselga $\mathbf{J}$ and Averbuch SD: ZD1839 ('Iressa') as an anticancer agent. Drugs 60: 33-40, 2000.

2. Shepherd FA, Rodrigues Pereira J, Ciuleanu T, et al: Erlotinib in previously treated non-small-cell lung cancer. $\mathrm{N}$ Engl J Med 2: 123-132, 2005.

3. Kris MG, Natale RB, Herbst RS, et al: Efficacy of gefitinib, an inhibitor of the epidermal growth factor receptor tyrosine kinase, in symptomatic patients with non-small cell lung cancer: a randomized trial. JAMA 16: 2149-2158, 2003.

4. Inoue A, Suzuki T, Fukuhara T, et al: Prospective phase II study of gefitinib for chemotherapy-naive patients with advanced nonsmall-cell lung cancer with epidermal growth factor receptor gene mutations. J Clin Oncol 21: 3340-3346, 2006.

5. Yun CH, Mengwasser KE, Toms AV, et al: The T790M mutation in EGFR kinase causes drug resistance by increasing the affinity for ATP. Proc Natl Acad Sci USA 6: 2070-2075, 2008.

6. Engelman JA, Zejnullahu K, Mitsudomi T, et al: MET amplification leads to gefitinib resistance in lung cancer by activating ERBB3 signaling. Science 5827: 1039-1043, 2007.

7. Guha U, Chaerkady R, Marimuthu A, et al: Comparisons of tyrosine phosphorylated proteins in cells expressing lung cancerspecific alleles of EGFR and KRAS. Proc Natl Acad Sci USA 37: 14112-14117, 2008

8. Arthur WT and Burridge K: RhoA inactivation by p190RhoGAP regulates cell spreading and migration by promoting membrane protrusion and polarity. Mol Biol Cell 9: 2711-2720, 2001.

9. Tikoo A, Czekay S, Viars C, et al: p190-A, a human tumor suppressor gene, maps to the chromosomal region 19q13.3 that is reportedly deleted in some gliomas. Gene 1: 23-31, 2000. 
10. Roof RW, Haskell MD, Dukes BD, Sherman N, Kinter M and Parsons SJ: Phosphotyrosine (p-Tyr)-dependent and -independent mechanisms of p190 RhoGAP-p120 RasGAP interaction: Tyr 1105 of p190, a substrate for c-Src, is the sole p-Tyr mediator of complex formation. Mol Cell Biol 12: 7052-7063, 1998.

11. Moran MF, Polakis P, McCormick F, Pawson T and Ellis C: Protein-tyrosine kinases regulate the phosphorylation, protein interactions, subcellular distribution and activity of p21ras GTPase-activating protein. Mol Cell Biol 4: 1804-1812, 1991.

12. Kusama T, Mukai M, Endo H, et al: Inactivation of Rho GTPases by 190 RhoGAP reduces human pancreatic cancer cell invasion and metastasis. Cancer Sci 9: 848-853, 2006.

13. Shen CH, Chen HY, Lin MS, et al: Breast tumor kinase phosphorylates p190RhoGAP to regulate rho and ras and promote breast carcinoma growth, migration and invasion. Cancer Res 19: 7779-7787, 2008.

14. Moon SY and Zheng Y: Rho GTPase-activating proteins in cell regulation. Trends Cell Biol 1: 13-22, 2003.

15. Costa DB, Halmos B, Kumar A, et al: BIM mediates EGFR tyrosine kinase inhibitor-induced apoptosis in lung cancers with oncogenic EGFR mutations. PLoS Med 10: 1669-1679, 2007.

16. Mitchell CE, Belinsky SA and Lechner JF: Detection and quantitation of mutant K-ras codon 12 restriction fragments by capillary electrophoresis. Anal Biochem 1: 148-153, 1995.

17. Forbes S, Clements J, Dawson E, et al: Cosmic 2005. Br J Cancer 2: 318-322, 2006.
18. Massarelli E, Varella-Garcia M, Tang X, et al: KRAS mutation is an important predictor of resistance to therapy with epidermal growth factor receptor tyrosine kinase inhibitors in non-smallcell lung cancer. Clin Cancer Res 10: 2890-2896, 2007.

19. Detterbeck FC, Boffa DJ and Tanoue LT: The new lung cancer staging system. Chest 1: 260-271, 2009.

20. Ma L, Liu YP, Geng CZ, Wang XL, Wang YJ and Zhang XH: Over expression of RhoA is associated with progression in invasive breast duct carcinoma. Breast J 1: 105-107, 2010.

21. Xiaorong L, Wei W, Liyuan Q and Kaiyan Y: Underexpression of deleted in liver cancer 2 (DLC2) is associated with overexpression of RhoA and poor prognosis in hepatocellular carcinoma. BMC Cancer 8: 205, 2008.

22. Dittert DD, Kielisch C, Alldinger I, et al: Prognostic significance of immunohistochemical RhoA expression on survival in pancreatic ductal adenocarcinoma: a high-throughput analysis. Hum Pathol 7: 1002-1010, 2008.

23. Takenawa T and Miki H: WASP and WAVE family proteins: key molecules for rapid rearrangement of cortical actin filaments and cell movement. J Cell Sci 10: 1801-1809, 2001.

24. Tsubakimoto K, Matsumoto K, Abe H, et al: Small GTPase RhoD suppresses cell migration and cytokinesis. Oncogene 15: 2431-2440, 1999.

25. Downward J: Targeting RAS signalling pathways in cancer therapy. Nat Rev Cancer 1: 11-22, 2003. 